\title{
Calculation of power spectra for block coded signals
}

\author{
Justesen, Jørn
}

Published in:

I E E E Transactions on Communications

Link to article, DOI:

10.1109/26.911442

Publication date:

2001

Document Version

Publisher's PDF, also known as Version of record

Link back to DTU Orbit

Citation (APA):

Justesen, J. (2001). Calculation of power spectra for block coded signals. I E E E Transactions on Communications, 49(3), 389-392. https://doi.org/10.1109/26.911442

\section{General rights}

Copyright and moral rights for the publications made accessible in the public portal are retained by the authors and/or other copyright owners and it is a condition of accessing publications that users recognise and abide by the legal requirements associated with these rights.

- Users may download and print one copy of any publication from the public portal for the purpose of private study or research.

- You may not further distribute the material or use it for any profit-making activity or commercial gain

- You may freely distribute the URL identifying the publication in the public portal

If you believe that this document breaches copyright please contact us providing details, and we will remove access to the work immediately and investigate your claim. 


\title{
Transactions Letters
}

\section{Calculation of Power Spectra for Block Coded Signals}

\author{
Jørn Justesen
}

\begin{abstract}
We present some improvements in the procedure for calculating power spectra of signals based on finite state descriptions and constant block size. In addition to simplified calculations, our results provide some insight into the form of the closed expressions and to the relation between the spectra and other properties of the codes.
\end{abstract}

Index Terms-Line codes, power spectra.

\section{INTRODUCTION}

$\mathbf{S}$ PECTRA for line codes defined by a finite number of states and constant block size $N$ were calculated in [1]. Examples of codes providing error control may be found in [2] and [3]. We shall be interested only in the case of independent input symbols which allows us to calculate the transition probabilities for the states. The actual mapping of the input blocks on the output blocks is not important.

One purpose of this paper is to use available symbolic computation tools to simplify the calculations. However, the explicit expressions also provide more information about the relationship between the structural details of the codes and the spectrum.

\section{OUtLINE OF CALCULATION OF SPECTRA FOR 4B3T CODES}

In this section we shall give an outline of the computational procedure by calculating the power spectrum of the well-known Franaszek MS-43 code. For this code $N=3$, and there are four states, for which the sums of the transmitted symbols are $\pm 1 / 2$ and $\pm 3 / 2$.

For independent, equally distributed input symbols, the transition matrix is

$$
P_{1}=\left[\begin{array}{cccc}
6 / 16 & 5 / 16 & 0 & 1 / 16 \\
6 / 16 & 6 / 16 & 5 / 16 & 3 / 16 \\
3 / 16 & 5 / 16 & 6 / 16 & 6 / 16 \\
1 / 16 & 0 & 5 / 16 & 6 / 16
\end{array}\right] .
$$

We may conclude from the symmetry of the code that the eigenvectors are either even or odd, and we may find the even vectors

Paper approved by E. Ayanoglu, the Editor for Communication Theory and Coding Application of the IEEE Communications Society. Manuscript received June 23, 2000.

The author is with the Department of Telecommunication, Technical University of Denmark, DK-2800 Lyngby, Denmark (e-mail: jju@tele.dtu.dk).

Publisher Item Identifier S 0090-6778(01)02164-X. and the associated eigenvalues from the matrix $P_{1}^{\prime}$, while the odd vectors and eigenvalues are found from $P_{1}^{\prime \prime}$, where

$$
P_{1}^{\prime}=\left[\begin{array}{cc}
7 / 16 & 5 / 16 \\
9 / 19 & 11 / 16
\end{array}\right] \quad P_{1}^{\prime \prime}=\left[\begin{array}{cc}
5 / 16 & 5 / 16 \\
3 / 16 & 1 / 16
\end{array}\right] .
$$

$P_{1}^{\prime}$ has eigenvalue 1 and the eigenvector gives the stationary distribution, $\left(p_{2}, p_{1}\right)=(5 / 14,9 / 14)$. From $P_{1}^{\prime \prime}$ we get the characteristic equation $z^{2}-3 / 8 z-5 / 128$, which provides a recursion for the correlation function $R_{1}(k)$ when values separated by multiples of $N=3$ are considered.

$$
R_{1}(k)=3 / 8 R_{1}(k-3)+5 / 128 R_{1}(k-6) .
$$

Thus when enough values of the correlation functions have been determined, we can express the series

$$
\sum_{-\infty}^{\infty} R_{1}(k) z^{k}
$$

as a rational function with denominator

$$
\left(1-3 / 8 z^{3}-5 / 128 z^{6}\right)\left(1-3 / 8 z^{-3}-5 / 128 z^{-6}\right) .
$$

The first step in calculating the correlation values is to find the average value of the correlation between two blocks

$$
r_{k}=E\left[c_{j} \# c_{j+k}\right]
$$

where we use the symbol \# to indicate a correlation. For blocks of length 3, we may write this as a vector of length 5. For $k=0$ this average has to be calculated from the actual list of codeblocks weighted by the stationary probabilities of the states

$$
r_{0}=\left[R^{\prime}(-2), R^{\prime}(-1), R_{1}(0), R^{\prime}(1), R^{\prime}(2)\right]
$$

where $R_{1}(0)$ is the actual average power, but $R_{1}(1)$ and $R_{1}(2)$ also have a contribution from the correlation between neighboring blocks. For the MS- 43 code we get

$$
r_{0}=\frac{1}{224}[-23 / 3,-82 / 3,145,-82 / 3,-23 / 3] .
$$

For $k>0$, we may simplify the calculations by first calculating the average value of each block. Let the average value of a block following state $i$ be $v_{a i}$. In this case

$$
v_{a 1}=(0,0,0) \quad v_{a 2}=(5 / 16,5 / 16,5 / 16) .
$$


The average value of the block preceding state $i$ is similarly found from the reverse transition matrix

$$
P_{1 r}=\left[\begin{array}{cccc}
3 / 8 & 5 / 24 & 5 / 48 & 1 / 16 \\
9 / 16 & 3 / 8 & 5 / 16 & 0 \\
0 & 5 / 16 & 3 / 8 & 9 / 16 \\
1 / 16 & 5 / 48 & 5 / 24 & 3 / 8
\end{array}\right] .
$$

In our case the averages are

$$
\begin{aligned}
& v_{b 1}=(-1 / 16,-1 / 16,-3 / 16) \\
& v_{b 2}=(-7 / 40,-7 / 40,-16 / 40) .
\end{aligned}
$$

We shall further need the quantities

$$
t_{i j}=p_{i} v_{b i} \# v_{a j}
$$

which in our case equal 0 for $j=1$, while for $j=2$ we get

$$
\begin{aligned}
t_{12} & =\frac{-45}{48 \cdot 224}(3,4,5,2,1) \\
t_{22} & =\frac{-10}{48 \cdot 224}(16,23,30,14,7) .
\end{aligned}
$$

We now get

$$
r_{1}=\left[R^{\prime \prime}(1), R^{\prime \prime}(2), R(3), R^{\prime}(4), R^{\prime}(5)\right]=\sum t_{i i}
$$

which gives us $R(3)$ and the remaining terms of $R(1)$ and $R(2)$

$$
R(k)=R^{\prime}(k)+R^{\prime \prime}(k)
$$

and further

$$
r_{2}=\left[R^{\prime \prime}(4), R^{\prime \prime}(5), R(6), R^{\prime}(7), R^{\prime}(8)\right]=\sum p_{i j} t_{i j}
$$

where $p_{i j}$ are the transition probabilities. If more values were needed, we could similarly calculate them from the $t_{i j}$ and higher order transition probabilities. However, in our case the block recursion can now be applied, and the recursion for individual correlation values applies from $R(9)$.

$$
\begin{aligned}
& R_{1}(1)=-23 / 168 \\
& R_{1}(2)=-299 /(24 \cdot 224) \\
& R_{1}(3)=-25 / 896 \\
& R_{1}(4)=-3715 /(3 \cdot 256 \cdot 224) \\
& R_{1}(5)=-3170 /(3 \cdot 256 \cdot 224) \\
& R_{1}(6)=-2625 /(3 \cdot 256 \cdot 224) \\
& R_{1}(7)=-14425 /(3 \cdot 2048 \cdot 224) \\
& R_{1}(8)=-11900 /(3 \cdot 2048 \cdot 224) .
\end{aligned}
$$

Up to this point only simple calculations have been needed. The remaining steps are conveniently performed by a tool for symbolic computation (actually an advanced calculator is sufficient). We express the tail of the correlation function as

$$
f(z)=\sum_{k \geq 3} R_{1}(k) z^{k}=-z^{3} g(z) /\left(1-3 / 8 z^{3}-5 / 128 z^{6}\right)
$$

and $g(z)$ is obtained by multiplying both sides of the equation by the denominator, since we know that the remaining correlation coefficients satisfy the recursion, and thus they do not contribute to $g(z)$.

$$
\begin{aligned}
g(z)= & \frac{25}{896}+\frac{3715}{172032} z+\frac{3170}{172032} z^{2}+\frac{825}{172032} z^{3} \\
& +\frac{205}{86016} z^{4}+\frac{1195}{688128} z^{5} .
\end{aligned}
$$

Finally we have the $Z$-transform of the correlation function as

$R_{1}(z)=\frac{145}{224}-\frac{23}{168}\left(z+z^{-1}\right)-\frac{299}{5336}\left(z^{2}+z^{-2}\right)+f(z)+f\left(z^{-1}\right)$.

Substituting $z=\exp (-\omega)$, we get the power spectrum.

$$
\begin{aligned}
S_{1}(\omega)= & \frac{1}{28} \frac{\operatorname{sn}(\omega)}{18713-11808 \cos 3 \omega-1280 \cos 6 \omega} \\
\operatorname{sn}(\omega)= & 348945-116902 \cos \omega-5843 \cos 2 \omega \\
& -236970 \cos 3 \omega+28264 \cos 4 \omega+6986 \cos 5 \omega \\
& -27600 \cos 6 \omega+2720 \cos 7 \omega+400 \cos 8 \omega .
\end{aligned}
$$

Note that there is a missing factor in the denominator of [1], and even when that is corrected, there appears to be other problems with the expression. However, the figure shows the correct spectrum.

\section{Relations Between the Choice of Code Blocks AND THE POWER SPECTRUM}

The fairly complex form of the spectrum calculated in the previous section can be traced back to the vectors $v_{b i}$ and $v_{a i}$. In the choice of code blocks, all vectors with a given sum are usually included. The only exceptions in this particular code are that the vectors $(1,1,-1)$ and $(-1,-1,1)$ are not used in states $\pm 1 / 2$. The vectors of sum \pm 1 therefore give different contributions to the three positions, and also contribute to the correlation in an irregular way. It turns out that the calculations are greatly simplified, and the result also much more intuitive, if we make the slightly artificial change of using the three words $(-1,1,1),(1,1,-1)$, and $(1,-1,1)$ with probability $2 / 3$. We are not proposing to change the code in this way, but to adopt this approximation in the calculation of the spectrum. Just as the choice of a particular block of a certain weight is rather arbitrary, we do not expect this approximation to have any important effect on the power spectrum.

The transition matrix is unchanged. We may now write all the average vectors as

$$
v_{a i}=u_{a i} e \quad v_{b i}=u_{b i} e
$$

where

$$
e=(1,1, \ldots, 1)
$$

and we only need to calculate the constants $u_{a i}$ and $u_{b i}$. These may be found as the average values of the original vectors, but they have a more intuitive interpretation as the average weight of a word before or after a given state. Next the $t_{i j}$ may be replaced by scalars which are all multiplied by the vector $e \# e=$ $(1,2, \ldots, N, \ldots, 1) / N$. These scalars determine $R(N k)$, and the remaining correlation values are obtained by linear interpolation since the decimated correlation sequence should be convolved with the sequence $e \# e$.

To complete the correlation function, we need to consider the vector (we let $N=3$ )

$$
r_{0}=\left[R^{\prime}(1) / 2, R^{\prime}(1), R(0), R^{\prime}(1), R^{\prime}(1) / 2\right] .
$$

We can bring this vector to the same form by subtracting $R(0)-$ $3 R^{\prime}(1) / 2$ from $R(0)$. This has the effect of subtracting a con- 
stant value from the spectrum (which makes the resulting terms negative). We proceed to write the sequence in $z$-notation, and convert the result to the spectrum

$$
S(\theta)=R(0)+R^{\prime}(0)-S^{\prime}(3 \theta)(3+4 \cos \theta+2 \cos 2 \theta) .
$$

Here $S^{\prime}(3 \theta)$ is a function that is closely related to the spectrum of the sum of each block, and it depends only on the transition matrix. There is a "pulse shaping" factor corresponding to constant blocks with the higher symbol rate, and finally a constant is added to make the zero at $\theta=0$.

In [4] we discussed power spectra which were determined by a zero in the numerator at $z=1$ and a pole for a real value $<1$. In particular we found that the width of the suppressed band was related to the value of the real pole. For low frequencies we have very similar properties for the spectra considered here, and we should expect better low-frequency suppression with a smaller eigenvalue. As we observed in the example, the largest eigenvalue of the difference matrix also has a major influence on the correlation function. Consider the difference matrix

$$
P^{\prime \prime}=\left[\begin{array}{cc}
5 & b \\
3 & a-4
\end{array}\right] / 16
$$

The eigenvalue is minimized by taking $b$ to be the smallest possible value, -2 . This choice favors transitions from state $1 / 2$ to $-3 / 2$ rather than $3 / 2$, which tends to keep the intermediate values of the sum closer to 0 .

In [2] convolutional codes are combined with spectrum shaping. Usually error-correcting codes leave the spectrum of the transmitted signal constant, but in these constructions, additional redundancy is used to introduce a spectral null at zero frequency. The state space of the code is the product of the states of the convolutional code and the set of values of the running sum. The authors suggest that the decoder should neglect the value of the sum in order to limit the complexity of the decoder. The examples of power spectra suggest that a change in the convolutional code has little effect on the power spectrum. Thus we shall take the complementary approach here and calculate an approximate power spectrum under the assumption that the effect of the convolutional code can be neglected.

Example 1-Spectrum of Trellis Code: We shall follow the example in [2, Section II], letting two input bits be encoded by a rate $2 / 3$ encoder, such that the three output bits determine a coset and the remaining four bits determine a block of four symbols to be transmitted. The alphabet is $\{ \pm 1, \pm 3\}$. We shall make the approximation that the coset is randomly chosen rather than being determined by the convolutional code. Under this assumption the 256 blocks are divided into two sets such that all blocks with positive weight are in set $S^{+}$and all that have negative weight are in $S^{-}$. The blocks of weight 0 are assigned in such a way that whenever $x$ is in $S^{+},-x$ is assigned to $S^{-}$. It is observed in [2] that assigning the blocks based on the sign of the first symbol leads to two rather different spectra. This is not surprising considering that this choice will have a significant impact on $R(1)$, and indirectly on other correlation coefficients. We shall base our calculation on an assignment that allows the simplified form of the power spectrum discussed in this section. The number of vectors of weight $12,10, \ldots, 0$ in $S^{+}$are 1,4 , $10,20,31,40,22$. These numbers scaled by $1 / 128$ provide the columns of the 12 by 12 transition matrix, but the calculations involve only the 6 by 6 even and odd parts. In particular we get the stationary distribution

$$
\begin{array}{r}
p=(0.004386,0.021930,0.065789, \\
0.153509,0.289474,0.464912)
\end{array}
$$

and the characteristic polynomial of the odd matrix

$$
\begin{aligned}
& z^{6}-0.53125 z^{5}+0.143555 z^{4}-0.019604 z^{3} \\
& \quad+0.001461 z^{2}-0.000043
\end{aligned}
$$

The average value of the next block (weight $/ N$ ) is readily found to equal $\pm 57 / 64$, while more effort is required to find the previous averages.

$$
\begin{aligned}
u_{b}= & (2.4844,1.9344,1.4344,0.9844,0.5677,0.1919) \\
t= & (0.010896,0.042421,0.094367 \\
& 0.15110,0.164337,0.089227)
\end{aligned}
$$

As in the previous example we can now calculate $R(N)$, $R(2 N), \ldots, R(6 N)$

$$
\begin{aligned}
R(4 k)= & (0.491943,0.112988,0.019674 \\
& 0.001206,-0.000546,-0.000224) .
\end{aligned}
$$

The average correlations within a block are

$$
r_{0}=(0,0,5,0,0)
$$

And setting $R^{\prime}(0)=0$, we can calculate the spectrum as shown in the equation at the bottom of the page. The result is a compromise between the two curves shown in [2] for specific choices of weight zero blocks.

\section{COMPARISON OF POWER SPECTRA}

In this section we shall give a few examples to demonstrate the dependence of the low-frequency suppression on the state transfer functions. We shall also discuss the quality of the approximation introduced in Section III.

$$
\begin{aligned}
S(\theta) & =5-S^{\prime}(3 \theta)\left(1+\frac{3}{2} \cos \theta+\cos 2 \theta+\frac{1}{2} \cos 3 \theta\right) \\
S^{\prime}(x) & =\frac{-0.283240+0.648729 \cos x-0.174686 \cos 2 x+0.032434 \cos 3 x-0.002766 \cos 4 x+0.000142 \cos 5 x}{0.651611-0.610357 \cos x+0.154180 \cos 2 x-0.020386 \cos 3 x+0.001484 \cos 4 x-0.000043 \cos 5 x}
\end{aligned}
$$




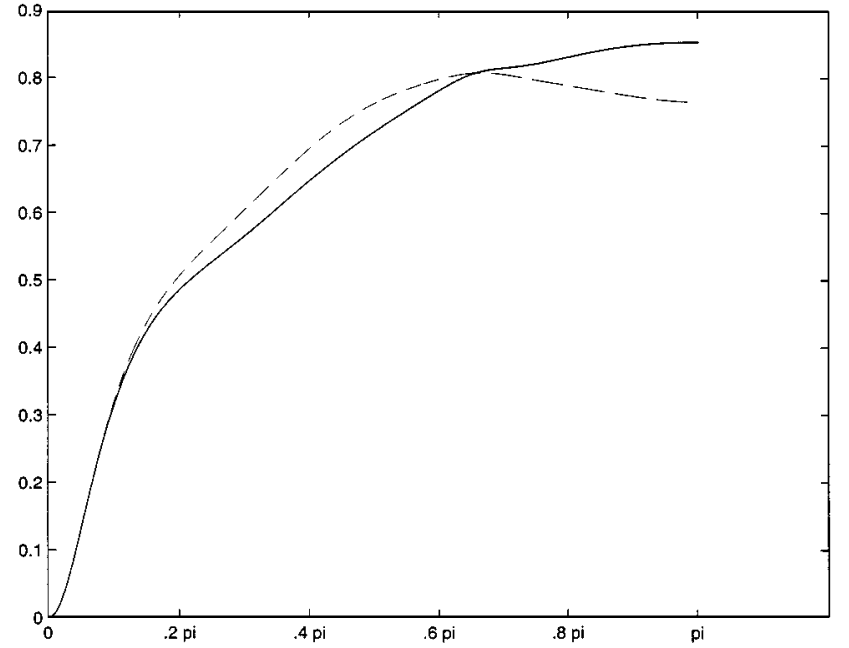

Fig. 1. Power spectrum of the MMS-43 code (solid) and an approximation (dashed).

Example 2-The MMS-43 Code: The design of this code for ISDN subscriber lines was based on reducing the so-called digital sum variation [5]. Following the approach from Section II, we may calculate the exact spectrum as

$$
\begin{aligned}
S(\theta)= & \frac{\operatorname{sn}(\omega)}{234260-126880 \cos 3-19968 \cos 6} \\
\operatorname{sn}(\omega)= & 154713-55172 \cos \omega-3419 \cos 2 \omega \\
& -92194 \cos 3 \omega+9820 \cos 4 \omega+1068 \cos 5 \omega \\
& -15328 \cos 6 \omega+768 \cos 7 \omega-256 \cos 8 \omega .
\end{aligned}
$$

In the MMS-43 code there are the four blocks of weight -1 in state $1 / 2$ and the corresponding vectors in state $-1 / 2$. The three permutations of $(0,0-1)$ are included, and as the last block $(-1,1,-1)$ is arbitrarily chosen. We may approximate the spectrum by using the three words $(-1,-1,1),(-1,1,-1)$, and $(1,-1,-1)$ with probability $1 / 3$. In this case we find the spectrum

$$
\begin{aligned}
S(\theta) & =21 / 26-S^{\prime}(\theta)\left(1+\frac{4}{3} \cos +\frac{2}{3} \cos 2\right) \\
S^{\prime}(\theta) & =\frac{137952-41384 \cos 3 \theta-2432 \cos 6 \theta}{208(4505-2440 \cos 3 \theta-384 \cos 6 \theta)} .
\end{aligned}
$$

Fig. 1 shows that the approximation is quite good, particularly at low frequencies.

Example 3-A Code with Better DC Suppression: Based on the analysis in Section III and the discussion in [4], we see that the magnitude of the largest eigenvalue $<1$ in $P$ should be minimized. We can maintain the sum matrix, and thus the stationary distribution, while we choose the blocks for the two middle states to reduce the eigenvalue. We may either take two code blocks with sum +1 or -2 for state $1 / 2$, and obtain the eigenvalue $5 / 16$, or we can go further and take three words of sum -2 , which reduces the eigenvalue to $3 / 16$. This structure is obtained by using the three words of weight -2 in state $1 / 2$. As the last

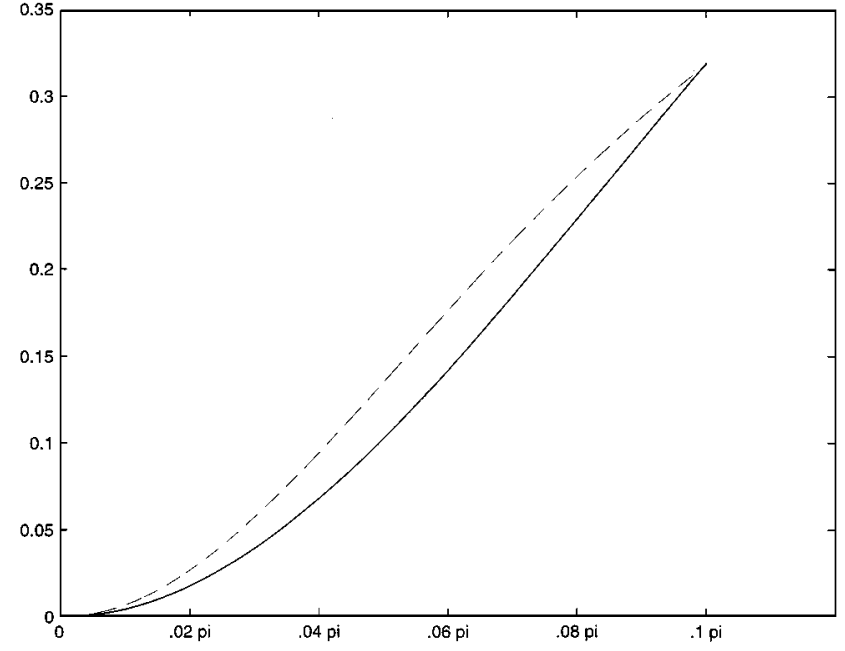

Fig. 2. Power spectra of the MMS-43 code (dashed) and a code with better low-frequency suppression (solid).

word we may take $(0,1,0)$, but we shall base the calculation of the power spectrum on the assumption that the three cyclic shifts are used with probability $1 / 3$. The resulting spectrum is

$$
\begin{aligned}
S(\theta) & =159 / 208-S^{\prime}(\theta)\left(1+\frac{4}{3} \cos \theta+\frac{2}{3} \cos 2 \theta\right) \\
S^{\prime}(\theta) & =\frac{272901+179176 \cos 3 \theta-13184 \cos 6 \theta}{208(17993-10480 \cos 3 \theta+768 \cos 6 \theta)} .
\end{aligned}
$$

Fig. 2 shows that the spectrum is lower than that of the MMS-43 code found in Example 2 for $\theta<0.1 \pi$.

\section{ConCLusions}

We have demonstrated that the power spectra of some wellknown codes can be calculated exactly by a modification of a commonly used numerical procedure. This approach not only reduces the amount of computation, but also gives some insight into the properties of a code which contribute to a desirable spectrum. Finally we suggest an approximate calculation which gives a more intuitive interpretation of the power spectra.

\section{ACKNOWLEDGMENT}

The author would like to thank R. Calderbank for making available his manuscript, which contains several related calculations.

\section{REFERENCES}

[1] G. L. Cariolaro and G. P. Tronca, "Spectra of block coded digital signals," IEEE Trans. Commun., vol. COM-22, pp. 1555-1563, Oct. 1974.

[2] A. R. Calderbank, T.-A. Lee, and J. E. Mazo, "Baseband trellis codes with a spectral null at zero," IEEE Trans. Inform. Theory, vol. 34, pp. 425-434, May 1988.

[3] A. R. Calderbank and J. E. Mazo, "Spectral nulls and coding with large alphabets," IEEE Commun. Mag., vol. 29, pp. 58-67, Dec. 1991.

[4] J. Justesen, "Information rates and power spectra of digital codes," IEEE Trans. Inform. Theory, vol. IT-28, pp. 457-472, May 1982.

[5] Deutsche Bundespost, "ISDN Spezifikation der Schittstelle $\mathrm{U}_{k: 0}$," Technische Richtlinie, Schicht 1, 1987. 